\title{
Conflits Armes Et Representativite Ethnique Au Nord-Kivu En Republique Democratique Du Congo
}

\author{
Tabin Lissendja Bahama \\ Politologue, Enseignant-Chercheur, Chef de Travaux \\ Université de Kisangani, République Démocratique du Congo
}

doi: 10.19044/esj.2017.v13n23p393 URL:http://dx.doi.org/10.19044/esj.2017.v13n23p393

\begin{abstract}
The goal pursued in the present reflection is to analyze and explain the relationship of cause and effect between the persistence of armed conflicts and the issue of ethnic representation in political institutions in North-Kivu in Democratic Republic of Congo. Since the 1990s, is observed a systematic use of ethnicity in the calculations of political, economic and social actors active in the province. In addition to the question of nationality of immigrants, access to land and overpopulation, the question of representation of ethnic groups arises with acuity and remains at the center of the attitudes and behaviour of political and security actors on the ground. The elections held in the country in 2006, leading to the no representativeness of the tutsi ethnic group, have reconfigured the dominant positions available since the advent of rebellions in the country. An attempt to maintain or regain their status as, gun violence has been used by stakeholders, leading to the reactivation of the other ethnic dormant for the construction of bands armed self-defence groups. The persistence of the conflict in North Kivu in recent years would be also linked to the violent struggle triggered by members of the tutsi ethnic group in order to ensure their representation in provincial institutions of North Kivu, central Government or even in other strategic positions in the country. This gives political and security logically motivated other ethnic local to replenish the armed groups in order to address the threat of domination that tries to impose the tutsi ethnic group in North-Kivu.
\end{abstract}

Keywords: representativeness, armed conflicts, armed groups, North Kivu, ethnic groups.

\section{Résumé}

Le but poursuivi dans la présente réflexion est d'analyser et d'expliquer le rapport de cause à effet entre la persistance des conflits armés 
et la question de représentativité ethnique au sein des institutions politiques au Nord-Kivu en République Démocratique du Congo. Depuis les années 1990, il s'observe un recours systématique à l'ethnicité dans les calculs politiques, économiques et sociaux de différents acteurs actifs dans la province. En marge de la question de nationalité des immigrés, d'accès à la terre et de surpopulation, la question de représentativité de groupes ethniques se pose avec acuité et demeure au centre des attitudes et comportements politiques et sécuritaires des acteurs sur le terrain. Les élections organisées dans le pays en 2006, ayant abouti à la non représentativité du groupe ethnique tutsi, ont reconfiguré les positions dominantes dont il disposait depuis l'avènement des rebellions dans le pays. Pour tenter de conserver ou retrouver leur statut d'autant, la violence armée a été utilisée par les acteurs concernés, aboutissant ainsi à la réactivation des autres groupes ethniques en sommeil pour la construction de bandes armés d'autodéfense. La persistance de la conflictualité au Nord-Kivu ces dernières années serait également liée à la lutte violente déclenchée par les membres du groupe ethnique tutsi afin d'assurer leur représentativité au sein des institutions provinciales du NordKivu, du gouvernement central voire dans d'autres postes stratégiques dans le pays. Cette donne politique et sécuritaire a logiquement motivé les autres ethniques locales à reconstituer les groupes armés afin de faire face à la menace de domination que tente d'imposer le groupe ethnique tutsi au NordKivu.

Mots clés : représentativité, conflits armés, groupes armés, Nord-Kivu, groupes ethniques.

\section{Introduction}

La crise politique qui jalonne l'histoire de la République Démocratique du Congo dont certaines ont dégénéré en conflits armés ou rébellion peut-elle être appréhendée dans l'optique de la lutte pour représentativité des ethnies locales ? Si tel n'est pas le cas sur l'ensemble du territoire national, l'analyse objective de la situation de l'Est du pays, et de la Province du Nord-Kivu, mérite une attention soutenue. La question de représentativité politique, mais surtout ethnique, demeure au cœur de la conflictualité au Nord-Kivu en RDC.

Pour Philippe Braud (2011), toute théorie du gouvernement représentatif est nécessairement confrontée au dilemme suivant : convient-il de représenter l'unité du groupe ou bien la diversité de ces composantes ? De Hobbes à Rousseaux, l'accent est placé sur la première conception. Les représentants ne sont pas les délégués de telle ou telle fraction de la population, ils incarnent l'ensemble du corps politique, ils en expriment la volonté générale. Ainsi, le corps représenté doit être considéré comme un 
ensemble unifié, fondé sur la seule existence du lien politique à l'exclusion de toute autre considération particulariste d'origine ethnique, de religion, de langue, de culture. Mais à l'opposé se trouve l'idée selon laquelle la représentation doit permettre l'expression sur la scène politique des conflits d'intérêts et d'aspirations qui résultent de la fragmentation de la société en classe et catégories sociales. Entre les deux conceptions, la seconde caractérise les comportements et attitudes des populations africaines souvent repliées vers les particularités géographiques et ethniques.

La RDC est plongée dans un cycle de violences armées quelques années seulement après son acception à l'indépendance. De la rébellion muleliste à ce jour, 1'univers politique congolais est régulièrement confronté à la contestation armée de l'autorité de l'Etat ou de ses dirigeants. Benoît Verhaegen (1969) a longuement expliqué le phénomène des rébellions au Congo à travers ses différents écrits. Après l'écrasement des rébellions de 1964 par l'armée du Colonel Joseph Mobutu, les poches de résistance ont persisté dans la partie Est du pays avec des attaques sporadiques contre les positions de l'armée gouvernementale ou de services de sécurité du pays (Amuri Misako FD, 2011). Il a fallu attendre le courant des années 1980 (élections législatives de 1982 et 1987) et le début des années 1990 (perspectives des élections générales proposées à la conférence nationale souveraine) pour assister à l'escalade de la violence armée perpétrée par les acteurs privés membres des communautés locales.

La lutte pour l'occupation des terres et l'épineuse question de nationalité de population d'expression rwandaise n'ayant jamais trouvée une réponse définitive par le pouvoir central zaïrois de l'époque, les communautés locales, mobilisées par les leaders locaux, avaient pris l'option de résoudre le différend à leur manière (Pabanel JP, 1991). A cela s'était ajoutée la question de représentativité politique et ethnique dans la perspective des élections générales annoncées dans les assises de la conférence nationale souveraine (Rusamira E, 2003). Pour ce faire, les membres des ethnies Hunde, Tembo, Havu et Nyanga s'étaient directement attaqués aux populations d'expression rwandophone (hutu et tutsi) dans les territoires de Masisi et de Walikale, populations considérées comme étrangères sur le sol congolais (Mashaury Kule Tambile, 1987). La prise du pouvoir par le Front Patriotique Rwandais (FPR) de Paul Kagamé et le déferlement de réfugiés hutu rwandais à l'Est du zaïre sont venus aggravés une situation déjà préoccupante en augmentant la capacité de nuisance de Hutu face non seulement aux ethnies locales qui les considéraient comme des étrangers, mais aussi contre les tutsi qui venaient de prendre le pouvoir au Rwanda.

La guerre de l'AFDL et du RCD dont les tutsi constituaient la branche armée, avait occasionné les massacres à grande échelle des réfugiés 
hutu rwandais présents sur le sol congolais. Vu l'absence prolongée de l'Etat dans cette partie du pays et soumises à la loi du dominant (tutsi), les communautés locales se sont senties dans l'obligation de s'organiser pour assurer leur propre protection et celle de leur milieu. D'où le recours à la violence comme moyen d'autodéfense et d'expression politique face à d'autres acteurs armés présents dans la région. Mais depuis la fin le 30 juin 2003, la lutte politique violente au Nord-Kivu s'est atténuée pour laisser place à la compétition politique normalisée par l'élection des gouvernants. Les groupes ethnique se sont ainsi lancés dans la mobilisation politique et électorale afin d'assurer leur victoire aux élections à venir. Du coup, la question de représentativité politique a refait surface. Mais les résultats des élections législatives nationales et provinciales de $2006^{59}$ ayant concrétisé l'écrasant échec du RCD-Goma et, par ricochet, de la communauté tutsi au Nord-Kivu a posé le problème de leur représentativité au sein des institutions politiques de la province (De Saint Moulin L, 2006).

Pourquoi les conflits armés se sont poursuis au Nord-Kivu après les élections organisées dans tout le pays en 2006? Comment expliquer la non représentativité du groupe ethnique tutsi dans les institutions politiques de la province? Existe-t-il un lien entre l'échec du groupe ethnique tutsi aux élections législatives de 2006 au Nord-Kivu et la relance des conflits armés dans la province ? L'échec de la communauté tutsi aux élections législatives de 2006 au Nord-Kivu associé à leur non représentativité au sein des institutions politiques serait l'élément déclencheur de la poursuite des conflits armés dans la province après les élections de 2006. Le principal facteur de l'échec électoral du groupe ethnique tutsi serait leur faible poids démographie au Nord-Kivu en comparaison aux autres groupes ethniques.

\section{Matériels et méthodes}

L'analyse des attitudes et comportements des acteurs sur le terrain est au centre de la présente réflexion en vue de mieux comprendre leur prise de positions politiques et sécuritaires sur le terrain. La compréhension et l'analyse de l'objet sous examen nous a imposé une perspective sociohistorique en vue de replacer la conflictualité du Nord-Kivu dans le contexte de sa genèse et son évolution jusqu'à sa manifestation actuelle. Les données présentées dans cette étude ont été récoltées dans les différents territoires de la province qui sont Beni, Luberon, Rutshuru, Nyiragongo, Masisi et Walikale mais plus particulièrement à Goma, capitale de la province et siège des institutions politiques du Nord-Kivu. Ainsi, les bibliothèques disponibles ont été visitées ainsi que les différents rapports

${ }^{59}$ Commission Electorale Indépendante, «Les résultats des élections législatives de 2006 dans la province du Nord-Kivu en République Démocratique du Congo, 2006. 
annuels du gouvernant de la province et du bureau de l'Assemblée Provinciale du Nord-Kivu. Les données récoltés par le truchement de la technique documentaire ont été complétées grâce aux interviews réalisées sur le terrain auprès des étudiants, les députés provinciaux, les agents du gouvernorat du Goma, les acteurs armés et ceux de la société civile du NordKivu. L'analyse qualitative de données récoltées a servi au traitement et à l'interprétation des informations ayant donné forme et sens à la présente réflexion.

\section{Nature des conflits ethniques au Nord-Kivu}

\section{Evolutions des conflits et jeu d'alliance}

La transplantation des populations rwandaises dans le Kivu par le colonisateur constitue le point de départ de la conflictualité au Nord-Kivu en République démocratique du Congo. Sur ceux-ci s'ajoutent d'autres immigrés ayant fui la chute du pouvoir coutumier tutsi en 1959 au moment des préparatifs de l'accession du pays à l'indépendant, le colonisateur belge étant pour beaucoup dans l'émergence rapide et souhaité de la communauté hutu en prévision de l'accession au pouvoir à l'indépendance. Le nombre élevé des immigrés a posé directement le problème d'accès à la terre en tant que ressources naturelles indispensables pour la survie des personnes. L'attitude et le comportement déplacés des immigrés vis-à-vis du pouvoir coutumier voire administratif ont suffi aux autochtones pour réclamer à haute voix le départ de ceux qu'ils considèrent comme des étrangers venus du Rwanda voisin. Ainsi la question foncière peut se résumer en quelques mots selon les propos de Mathieu et Kazadi (1990):

- Rétrécissement de l'espace disponible pour des paysanneries de plus en plus nombreuses;

- Dépossession foncière de ces mêmes paysanneries, en grande partie organisée par la collusion entre chefs coutumiers, bourgeoisies urbaines et administrations corrompues;

- Incertitude et précarité croissantes des droits fonciers paysans, résultant à la fois des pratiques foncières clientélistes et opportunistes des chefs coutumiers autochtones et de la disqualification des droits fonciers traditionnels par les lois foncières modernes (promulguées en 1966 et 1973), au nom de la propriété étatique du sol.

La course engagée à la conquête du pouvoir politique par les immigrés se considérant comme de congolais/zaïrois à part entière en 1982 et 1987 peut être considérée comme le point de départ de la manifestation violente du conflit entre autochtones et immigrés au Nord-Kivu. Les conflits autour de l'accès à la terre constituent toujours un facteur crucial, et l'ouverture de la transition a ravivé la compétition politique. Avec la question de la représentation à la Conférence Nationale ouverte à Kinshasa en 1991, la 
volonté d'exclure les Banyarwanda de la nationalité congolaise et donc de l'exercice des droits politiques se manifeste à nouveau. Il faut bien sûr, là encore, tenir compte de l'extrême affaiblissement du Président Mobutu qui, sans doute, lui ôte dans une large mesure la maîtrise politique et militaire des conflits qui surgissent dans le pays mais qui peut également l'inciter à laisser se développer conflits et violences aux fins de démontrer que, sans lui, le pays est voué au chaos ${ }^{60}$.

L'effet de contagion des évolutions en cours au Rwanda, depuis que les Tutsis du Front Patriotique Rwandais (FPR) ont lancé en 1990 leur offensive de reconquête du pouvoir à partir de l'Ouganda, joue également un rôle déterminant. Koen Vlassenroot et Raeymaekers (2004), qui ont analysé le processus de développement en 1993 des luttes interethniques dans le Masisi, relève que l'un des facteurs de l'aggravation des tensions est l'encouragement que leur apporte la présidence hutu du Rwanda, dans le but de couper les liens que nouent les tutsi locaux et le FPR. La guerre du Rwanda puis l'arrivée des réfugiés hutu vont modifier le caractère des conflits socio-ethniques au Kivu : à des affrontements qui restaient localisés entre autochtones et Banyarwanda va se substituer une coalition rassemblant hutu du Kivu et réfugiés hutu, parfois rejoints par des milices autochtones, contre les tutsi congolais, dans un conflit de dimension régionale, imbriquant le Kivu du nord au sud et le Rwanda (Koen Vlassenroot et Raeymaekers, 2004).

Il s'observe donc que les tutsi congolais se sentant menacés au Congo par les autochtones avaient jugé nécessaire et utile d'aller appuyer leurs confrères ayant évolué en Ouganda dans les offensives militaires lancées contre le pouvoir hutu au Rwanda dirigé par le Président Juvénal Habyarimana. Cette alliance et coalition des tutsi congolais avec ceux de l'Ouganda contre les hutu du Rwanda était perçue comme une grande trahison par les hutu congolais vivant au Zaïre de l'époque. Avec la prise du pouvoir par les FPR au Rwanda en 1994 et le déferlement des réfugiés hutu rwandais au Zaïre qui étaient accueillis non seulement par le régime politique Mobutu mais aussi par leurs confrères hutu du Zaïre qui vivent au Kivu depuis de très longues années. Ensemble, ils auraient constitué un front commun pour s'attaquer aux tutsi présents au Zaïre et à leurs intérêts particuliers, occasionnant par conséquent, la fuite de beaucoup d'entre eux au Rwanda pour se mettre à l'abri. Leur installation le long des frontières entre le Zaïre d'une part, le Rwanda et le Burundi d'autre part, avait

60 Théorie de la décharge présentée par Achille Mbembe selon laquelle les autorités étatiques peuvent laisser pourrir une situation sociale facilement gérable en raison des intérêts et profits politiques qu'ils peuvent en tirer. Soit pour justifier leur omniprésence et indispensabilité pour la marche du pays, soit pour laisser l'espace d'action à leurs complices politiques et acteurs privés. 
constitué une véritable opportunité politique pour les acteurs régionaux d'intervenir à l'Est du Zaïre pour des raisons qu'ils qualifiaient de purement sécuritaire.

\section{Régionalisation du conflit}

Avec le déferlement des réfugiés hutu rwandais au Zaïre occasionné par la prise du pouvoir politique au Rwanda par les tutsi, une hostilité antitutsi s'installe au Zaïre incitant même le régime Mobutu à remettre en cause les acquis de leur nationalité zaïroise, y compris pour les Banyamulenge, les plus anciennement installés. En effet, «le 28 avril 1995, le parlement zaïrois adopta une série de résolutions destinées à assimiler tous les Zaïrois d'origine rwandaise, y compris les Banyamulenge, à des réfugiés et, par conséquent, à leur réserver le même traitement. Car les résolutions laissent entendre que les Banyamulenge (Zaïrois tutsi d'origine rwandaise) ont acquis la nationalité zaïroise de manière frauduleuse et appellent à leur expulsion, à l'annulation des contrats de propriété et à l'interdiction de leurs associations») (Pourtier R, 1999). Les Banyamulenge se sentent menacés par la mise en œuvre de ces mesures, prétexte de prendre les armes et déclencher une rébellion contre le gouvernement zaïrois le 13 octobre 1996 au Kivu.

En 1996, quatre catégories d'affrontements armés font rage au Kivu qui sert de base arrière aux guérillas des pays voisins en vue, selon eux, de rétablir la sécurité à leurs frontières. Ils opposent: les forces armées rwandaises (à dominance tutsi) aux anciens génocidaires hutu; les forces armées burundaises (à dominance tutsi) à ses adversaires extrémistes hutu; le gouvernement ougandais à deux groupes rebelles différents (les ADF/NALU et les LRA) ; enfin, un certain nombre d'organisations rebelles au gouvernement zaïrois. Les deux compagnons d'armes, le général et dirigeant du FPR Paul Kagamé et le Président ougandais Yoweri Museveni saisissent l'opportunité de cette rébellion et la soutiennent militairement pour faire pénétrer leurs troupes au Kivu. En fait, ils préparent le conflit depuis plusieurs mois, avec la participation du Burundi, pour plusieurs raisons. Ils font essentiellement valoir le caractère sécuritaire de leur engagement auprès de leurs frères tutsi Banyamulenge (Pourtier R, 1997). Cette régionalisation des conflits ou, pour d'autres, cette transplantation des conflits ethniques rwandais, burundais et ougandais au Zaïre avaient joué un rôle direct et indirect dans les logiques d'intervention et agissements de rebelles et autres groupes armés présents au pays.

Outre les préoccupations sécuritaires du Rwanda et de l'Ouganda, des ambitions politiques et économiques sous-tendent leur engagement auprès des Banyamulenge, qui s'inscrivent dans une stratégie globale : renverser le régime Mobutu, s'arroger un contrôle politique sur l'Est du pays et tirer profit des richesses naturelles de la région. C'est la matérialisation de ces visées 
politiques, économiques et stratégiques par les acteurs régionaux qui jettera la flamme dans la poudrière à l'Est de la RDC en général et au Nord-Kivu en particulier. Les soutiens mutuels et oppositions circonstancielles des groupes ethniques hutu et tutsi aux différents régimes politiques au Rwanda et au Zaïre par des zaïrois/congolais interposés seraient 1'un des facteurs à la base de la régionalisation des conflits, leur persistance et l'escalade de la violence armée dans cette région riche de la République Démocratique du Congo.

\section{Escalade de la violence et radicalisation des esprits}

La persistance des conflits ethniques au Kivu envenimée par le jeu d'alliance et de coalition entre groupes ethniques ayant des intérêts communs à sauvegarder ont débouché sur l'escalade de la violence avec recours systématique à l'arme à feu, contrairement à l'usage de l'arme blanche souvent utilisée par les groupes communautaires. Cette escalade de la violence a atteint le point culminant avec l'entrée en scène de l'AFDL (Alliance des forces démocratiques de libération du Congo) et de ses alliés fin octobre 1996. On observe ici un cas de conflit complexe et «multidimensionnel» Pourier R, 1999).

En octobre 1996, plus d'un million de réfugiés rwandais hutu est pris en tenaille dans des combats visant à démanteler leurs camps situés au Kivu. Quelques 600000 d'entre eux tentent d'échapper aux combats en rentrant au Rwanda. L'ONG Human Rights Watch (1997) témoigne de cette catastrophe humanitaire : « Dans certains camps, les ex-FAR et les milices ont opéré un retrait en hâte, parfois après avoir brièvement résisté à l'avancée de l'AFDL. En novembre 1996, la majorité de la population des camps, -peut-être 600 000 sur les 1,1 million que les camps auraient, selon les estimations, abrités en octobre 1996-, sont rentrés au Rwanda. Plusieurs milliers de personnes seraient mortes dans les premières semaines des attaques dans les camps, prises au milieu de tirs croisés entre l'AFDL et les ex-FAR, les milices et les FAZ; tuées par les anciennes autorités des camps afin d'empêcher leur retour au Rwanda, ou de les forcer à accompagner les ex-FAR et les miliciens dans leur retraite vers l'Ouest; ou encore tuées par les soldats de l'AFDL et de l'Armée patriotique rwandaise (APR) »

A la fin de l'année 1997, Laurent Désiré Kabila qui avait pris le pouvoir au mois de mai de la même année, avait commencé à prendre ses distances avec ses "protecteurs" rwandais et ougandais dont les troupes étaient stationnées au Kivu depuis son arrivée au pouvoir. En mai 1998, il les accuse de piller les ressources minières et agricoles de l'est du pays, et de porter atteinte à l'intégralité territoriale de la RDC en occupant la zone frontalière du Kivu. Il dénonce, en particulier, la volonté expansionniste du Rwanda vers cette région qui devient la véritable poudrière d'un conflit régional larvé. Les objectifs des nouveaux alliés se 
dessinent clairement : non seulement sécuriser les frontières pour empêcher les infiltrations de rebelles venus de la RDC vers leur pays respectif, mais aussi renverser Kabila devenu imprévisible et trop menaçant pour eux. De surcroît, et surtout, il s'agit de consolider leur mainmise sur la prédation fabuleuse des richesses naturelles de l'est de la RDC. Ainsi se dessine une nouvelle offensive de la coalition tutsi au pouvoir en Ouganda, au Rwanda et au Burundi en se servant, comme par le passé, des auxiliaires congolais en quête de pouvoir politique ou ayant de compte à rendre avec le nouveau pouvoir de Laurent Désiré Kabila. Avec les alliances nouées par le Président Laurent Désiré Kabila avec l'Angola, le Zimbabwe, la Namibie, la Zambie, le Tchad et la Lybie, la guerre s'est enlisée pour se terminer officiellement par l'entrée en vigueur du gouvernement de transition avec la formule $1+4$, avec Joseph Kabila, arrivée à la tête du pays en remplacement de Laurent Désiré Kabila assassiné dans des circonstances non encore clairement élucidées.

Avec ces guerres successives et les massacres à grande échelle perpétrés par les différents acteurs impliqués dans les conflits au Kivu, les esprits se sont radicalisés dans une crispation pas facile à désamorcer. En effet, pour la communauté tutsi en RDC comme pour le pouvoir tutsi au Rwanda, les hutu installés en RDC sont des génocidaires et doivent répondre de leurs actes devant la justice rwandaise ou internationale. Pourtant parmi les réfugiés hutu actuellement en âge adulte en $\mathrm{RDC}$, rares et très rares sont ceux ayant pris une part active dans le génocide de 1994. Une assimilation abusive ne peut être que construction politique afin de servir de justification de la présence de l'armée rwandaise sur le sol congolais. Pour les hutu rwandais basés en RDC, l'intransigeance du pouvoir actuel au Rwanda traduit l'absence de volonté politique afin de résoudre les différends par le dialogue. Dans cette configuration, seule la lutte armée demeure l'option sur la table pour contrer le pouvoir tutsi au Rwanda à tenir compte de préoccupations légitimes des réfugiés hutu rwandais.

Du côté des communautés congolaises du Nord-Kivu, l'attitude belliqueuse affichée par les tutsi congolais à travers le recours systématique à la violence comme moyen d'expression politique et leurs allégeances réelle ou supposée au pouvoir tutsi du Rwanda, favorisent leur assimilation aux occupants rwandais. Prétextant qu'ils ne sont pas en sécurité au Congo, ils doivent alors retourner au Rwanda afin de bénéficier de la sécurité que leur procure le pouvoir actuel tutsi au Rwanda ${ }^{61}$. Le refus de communautés

\footnotetext{
${ }^{61}$ Entretien avec deux membres de la société civile du Nord-Kivu à Goma, le 21 août 2014 ainsi que trois membres de la société civile de Béni, le 19 juillet 2015. Il y a lieu de noter que parmi les acteurs de la société civile du Nord-Kivu, il est bien difficile de trouver les membres de la communauté tutsi. Qu'il s'agisse de la ville de Goma, de Butembe et de Beni,
} 
locales d'un éventuel retour des «réfugiés » congolais qui seraient au Rwanda et en Ouganda depuis le déclenchement de la guerre de l'AFDL rentre dans cette logique de radicalisation des esprits où les groupes communautaires sont peu disposés à une cohabitation pacifique. Pour les communautés locales du Nord-Kivu, un tel retour risque de raviver les tensions ethniques à travers le rebondissement de l'épineuse question de l'accès à la terre ${ }^{62}$. La persistance des conflits ethniques dans le Masisi comme dans d'autres territoires du Petit Nord est à classer dans la quête des tutsi congolais à vouloir récupérer leurs terres légalement acquises auprès des autorités compétentes en matière d'acquisition des terres. Mais pour les communautés locales, il s'agit des terres ancestrales appartenant, depuis toujours, aux membres des groupes ethniques dits autochtones.

\section{Résultats}

\section{Configuration politique et rapport de force des acteurs au Nord-Kivu}

Depuis l'entrée en vigueur du gouvernement de transition en juin 2003 et la mise en route du processus électoral en RDC, les membres de la communauté tutsi du pays ont vu leur influence décroître sensiblement. En effet, il convient de rappeler qu'avec les rébellions de l'AFDL et surtout celle du RCD, plusieurs acteurs politiques et économiques tutsie congolais occupaient de postes stratégiques au sein de l'administration rebelle et des avantages économiques importants dans les zones sous contrôle rebelle. Mais depuis la réunification du pays en 2003 et la transformation des mouvements rebelles en partis politiques (Nkoko Lipambala J, 2009), les acteurs politiques tutsi inféodés dans la vie politique nationale avaient perdu une bonne partie de leur influence politique et stratégique.

Au niveau de la province du Nord-Kivu, l'organisation des élections législatives nationales et provinciales ont davantage affaibli l'aura des membres de la communauté tutsi sur le plan de la représentativité politique dans les instances politiques de la province. La configuration actuelle de l'univers politique du Nord-Kivu laisse entrevoir une forme d'exclusion de la communauté tutsi dans la gestion politique de la province en termes de représentativité. Les données récoltées sur le terrain sont présentées et analysées dans les points qui suivent en guise des résultats de l'étude.

les acteurs de la société civile sont à majorité les membres du groupe ethnique Yira du Grand-Nord.

${ }^{62}$ Les membres de la communauté tutsi ayant fui les affrontements pour se mettre à l'abri au Rwanda risquent de poser le problème de récupération de leurs terres acquises légalement auprès des autorités coutumières, administratives locales, provinciales ou centrales. Or, ces terres ont été réoccupées par les membres de communautés locales se disant réhabilités dans leurs droits de propriétaires terriens conformément à la coutume locale. 


\section{Représentativité ethnique au sein de l'Assemblée Provinciale}

Les guerres qui se déroulent actuellement dans plusieurs régions du monde sont qualifiées de guerres ethniques ou identitaires opposant des groupes ethniques à d'autres dans un contexte de l'exclusion. L'Afrique offre plusieurs exemples de ces types de conflits armés, particulièrement la région des grands lacs où le recours à l'ethnicité constitue une ressource politique efficace des acteurs pour se hisser au sommet de la société. Le recours à l'identité leur permet d'obtenir de position favorable dans la société en se servant de leurs groupes ethniques qu'ils prétendent représenter. «Forger par l'anthropologie pour analyser les sociétés primitives, cette notion de l'ethnicité s'est étendue, de façon mal maitrisée, à la science politique. Elle est utilisée soit dans une perspective développementaliste ou post-développementaliste afin de décrire le processus de construction étatique et nationale ainsi que les résistances qui les accompagnent, soit en politique comparée afin d'appréhender les modes d'intégration des minorités dans les systèmes politiques » (Hermet $\mathrm{G}$ et alii, 2010). Le recours à l'ethnicité en politique s'est accru depuis l'affaiblissement du régime Mobutu et la monté en puissance de ce que les Zaïrois de l'époque considéraient comme « géopolitique ${ }^{63}$

«Dans ce dernier usage, le plus fréquent, la notion d'ethnie rejoint celle de minorité culturelle. Dans l'un comme dans l'autre cas, elle peut présupposer que les communautés politiques doivent avoir un fondement ethnique, ce qui tend à faire de l'ethnicité une des composantes tant de la mobilisation politique que de la construction des systèmes politiques, et à conduire ainsi jusqu'à l'extrême que constitue l'épuration ethnique. Dans une perspective moins radicale, elle vise à restaurer le sentiment de fierté de groupes qui se sentaient humiliés et à donner une légitimité à leurs revendications. Son usage se retrouve de plus en plus dans les relations internationales, correspondant à la nature des nouveaux conflits internationaux. Comme tel, il se trouve partagé entre interprétation " ethnoréaliste ", qui en fait un paramètre nouveau de la géopolitique et une interprétation constructiviste qui souligne son rôle comme instrument de mobilisation politique » (Amselle JL et Elikia Mbokolo, 1985).

$\mathrm{Au}$ Nord-Kivu, le recours à l'identité est une stratégie politique fréquemment utilisée par les acteurs locaux, nationaux et régionaux pour un positionnement politique avantageux. En tant qu'instrument de mobilisation

\footnotetext{
${ }^{63}$ Avec l'instrumentalisation et la manipulation politicienne vers la fin du régime Mobutu, le terme «géopolitique » a été abusivement utilisé comme synonyme d'exclusion des non originaires d'une région de la politique locale. Cette conception de la géopolitique a donné lieu, entre 1991 et 1994, aux expulsions des non originaires des provinces ou régions où ils étaient pourtant solidement installés, en perdant ainsi tous ceux qu'ils avaient accumulés comme patrimoines.
} 
politique, l'ethnicité reste au Nord-Kivu un recours important tant pour la conquête démocratique que violente du pouvoir au niveau provincial que local. Lors des élections présidentielles, les législatives nationales et provinciales de 2006, l'ethnicité a été mobilisée par tous les acteurs politiques pour l'encadrement des électeurs membres de leurs communautés respectives en vue d'une victoire parfaite et d'une réelle représentation au niveau du pouvoir provincial et national (Lissendja Bahama T, 2010). Les groupes armés nationaux, pour leur part, recourent forcement à l'ethnicité pour la construction de leur mouvement, le recrutement des combattants et la confidentialité des opérations à mener sur le terrain.

A l'issue de législatives provinciales de 2006 au Nord-Kivu, 1'Assemblée provinciale a été constituée par les députés élus. Lors de la compétition électorale, chaque groupe ethnique avait mobilisé ses membres afin d'assurer sa représentativité au sein des instances politiques provinciales. Le tableau qui suit présente la situation des députés provinciaux du Nord-Kivu membres de l'Assemblée Provinciale.

Tableau 1 : Liste actualisée des députés provinciaux du Nord-Kivu

\begin{tabular}{|c|c|c|c|}
\hline $\begin{array}{l}\text { Circonscription } \\
\text { électorale }\end{array}$ & $\begin{array}{c}\text { Parti/Groupement } \\
\text { politique } \\
\text { d'appartenance }\end{array}$ & Noms de l'élu & $\begin{array}{l}\text { Groupes } \\
\text { ethniques }\end{array}$ \\
\hline \multirow[t]{3}{*}{ Ville de Goma } & Indépendant & Hon. Nzchanzchu Kende Kende & Nande \\
\hline & PPRD & Hon. Muhanya Bwinika Michel & Mushi \\
\hline & $\mathrm{RCD}$ & $\begin{array}{l}\text { Hon. Ushindi Kyalondawa Jean } \\
\text { Pierre }\end{array}$ & Rega \\
\hline $\begin{array}{ll}\text { Territoire } & \text { de } \\
\text { Nyiragongo } & \end{array}$ & PPRD & $\begin{array}{lll}\text { Hon. } & \text { Mutuele } & \text { Moromoro } \\
\text { Mutuel } & & \\
\end{array}$ & Hunde \\
\hline \multirow[t]{2}{*}{ Ville de Beni } & $\begin{array}{l}\text { Forces } \\
\text { renouveau }\end{array}$ & $\begin{array}{lll}\text { Hon. } & \text { Lukumbaka } & \text { Kyambi } \\
\text { Désiré } & & \\
\end{array}$ & Nande \\
\hline & PPRD & Hon. Mayombo Omari Jean & $\begin{array}{l}\text { Kusu } \\
\text { (suppléant) }\end{array}$ \\
\hline \multirow[t]{9}{*}{ Territoire de Beni } & Forces du renouveau & $\begin{array}{l}\text { Hon. Jaribu Muliwanyo Jean } \\
\text { Bosco }\end{array}$ & Nande \\
\hline & PPRD & $\begin{array}{l}\text { Hon. Kasereka Wanzavalere } \\
\text { Timothée }\end{array}$ & Nande \\
\hline & COFEDEC & $\begin{array}{lll}\text { Hon. } & \text { Kahindo } & \text { Tsekanabo } \\
\text { Janvier } & & \\
\end{array}$ & Nande \\
\hline & Forces du renouveau & Hon. Kavira Kananga Felly & Nande \\
\hline & RCD & Hon. Kule Kahandi Jérémie & Nande \\
\hline & MWAMI & Hon. Massali Kapula Liévin & Nande \\
\hline & COFEDEC & $\begin{array}{l}\text { Hon. Mathe Syndikiminya } \\
\text { Jéconie }\end{array}$ & Nande \\
\hline & MSR & $\begin{array}{lll}\text { Hon. Muntiki } & \text { Baumbilia } \\
\text { Kisombi Jean } & \\
\end{array}$ & Nande \\
\hline & PPRD & $\begin{array}{llll}\text { Hon. Kasereka Giza Jean de } \\
\text { Dieu }\end{array}$ & Nande \\
\hline Ville de Butembo & Indépendant & Hon. Kakule Lukambo Maombi & Nande \\
\hline
\end{tabular}




\begin{tabular}{|c|c|c|c|}
\hline & Forces du renouveau & $\begin{array}{lll}\text { Hon. } & \text { Muhahirwa } & \text { Kazungu } \\
\text { Simon } & & \\
\end{array}$ & Nande \\
\hline & Forces du renouveau & Hon. Paluku Kataka Emery & Nande \\
\hline \multirow{8}{*}{$\begin{array}{l}\text { Territoire } \quad \mathrm{de} \\
\text { Lubero }\end{array}$} & UPRDI & Hon. Butsapu Ndungo Haggaï & Nande \\
\hline & Mwami & Hon. Muhindo Nzanzu Bonané & Nande \\
\hline & PPRD & Hon. Kanzuli Kiezele Benito & Nande \\
\hline & PDC & Hon. Katembo Kabuyaya Domi & Nande \\
\hline & Forces du renouveau & $\begin{array}{ll}\text { Hon. Kayisavesa } & \text { Mbake } \\
\text { Wighong } & \end{array}$ & Nande \\
\hline & MSR & Hon. Maghuta Kamwenge & Nande \\
\hline & MSR & Hon. Muhindo Nzangi Butondo & Nande \\
\hline & Forces du renouveau & $\begin{array}{lll}\text { Hon. } & \text { Mukasasenge } & \text { Fataki } \\
\text { Pascal } & & \\
\end{array}$ & Nande \\
\hline \multirow{7}{*}{$\begin{array}{l}\text { Territoire } \quad \mathrm{de} \\
\text { Masisi }\end{array}$} & $\mathrm{RCD}$ & Hon. Habinshuti Seninga Robert & Hunde \\
\hline & MWAMI & $\begin{array}{l}\text { Hon. Bahati Kaembe Maphumo } \\
+\end{array}$ & Hunde \\
\hline & MLC & Hon. Banda Mulera Bernard & Hutu \\
\hline & PDC & Hon. Mulemeri Pilipili Abdoul & Hunde \\
\hline & PPRD & Hon. Nkuba Kahombo Sylvestre & Hunde \\
\hline & PANADI & $\begin{array}{l}\text { Hon. Sebishimbo Rubuga Jean } \\
\text { Bosco }\end{array}$ & Hutu \\
\hline & $\mathrm{RDC}$ & $\begin{array}{lll}\text { Hon. } & \text { Sebujangwe } & \text { Kirivita } \\
\text { Bertin } & & \\
\end{array}$ & Hutu \\
\hline \multirow[t]{3}{*}{$\begin{array}{ll}\text { Territoire } & \text { de } \\
\text { Walikale } & \end{array}$} & MSR & $\begin{array}{l}\text { Hon. Bwira Shemusimiwa } \\
\text { Manassé }\end{array}$ & Nyanga \\
\hline & Mwami & Hon. Eliba Omba Baudouin & Nyanga \\
\hline & PPRD & Hon. Kalinda Kahombo Gilbert & Nyanga \\
\hline \multirow{6}{*}{$\begin{array}{ll}\text { Territoire } & \text { de } \\
\text { Rutshuru } & \end{array}$} & COFEDEC & Hon. Bariyanga Rutuye Léon & Hutu \\
\hline & RCD & $\begin{array}{l}\text { Hon. Hakizumwami Habimana } \\
\text { Jules }\end{array}$ & Hutu \\
\hline & PPRD & $\begin{array}{l}\text { Hon. Kunemutumba Mbakwa } \\
\text { Samuel }\end{array}$ & Nande \\
\hline & $\mathrm{RCD}$ & $\begin{array}{l}\text { Hon. Mizerero Sebirande } \\
\text { Déogratias }\end{array}$ & Hutu \\
\hline & $\mathrm{RCD}$ & $\begin{array}{ll}\text { Hon. } & \text { Nyagashende } \\
\text { Munyazikweye } & \text { Bercky }\end{array}$ & Hutu \\
\hline & MSR & Hon. Simisi Nzala Zabulon & Nande \\
\hline
\end{tabular}

Source : Rapport annuel de l'Assemblée Provinciale du Nord-Kivu, décembre 2014, actualisé.

Le présent tableau indique la représentativité de groupes ethniques au sein de la plus haute instance politique de la province qu'est l'Assemblée Provinciale. La lecture du tableau montre que sur les 42 députés élus pour constituer l'Assemblée Provinciale, le groupe ethnique Nande représente, à lui seul, 24 députés provinciaux, soit 57, 14\% de l'effectif total de l'organe délibérant de la province. Un véritable poids politique qui avait permis, en janvier 2007, au candidat Julien Paluku, membre de ce groupe ethnique, de 
briguer le poste du Gouverneur de province à l'issue des élections organisées au second degré. Dans cette configuration de l'Assemblée provinciale, il est fort possible qu'en cas d'organisation de nouvelles élections au poste du Gouverneur, si jamais le nouveau maître de la province venait de démissionner ou d'être destitué, que son successeur soit encore membre du groupe ethnique Nande et ce, au regard du recours systématique à l'ethnicité comme instrument de mobilisation politique dans la province.

La deuxième force politique est constituée du groupe ethnique Hutu qui compte 7 députés provinciaux au sein de l'Assemblée provinciale du Nord-Kivu, soit $16,66 \%$. Cette représentation a permis à ce groupe ethnique d'avoir non seulement de représentants au niveau du gouvernement provincial du Nord-Kivu mais aussi de briguer le poste du Président de l'Assemblée provinciale. La troisième force politique au sein de l'Assemblée provinciale est constituée des membres du groupe ethnique Hunde, avec 5 députés élus, soit 11,90\%. Après vient le groupe ethnique Nyanga de Walikale avec 3 députés provinciaux élus, soit 7,14\%. Enfin, il y a 1 Mushi du Sud-Kivu et 1 Lega du Maniema élus dans la circonscription électorale de la ville de Goma et 1 Kusu de Walikale élu dans la circonscription électorale de la ville de Beni, représentant chacun 2,38\%.

En effet, 17 groupes ethniques peuplent la province du Nord-Kivu. Cependant, d'autres groupes ethniques semblent peu connus, ils constituent surtout le sous-groupe de la grande communauté Yira vivant dans le Grand Nord. Il s'agit particulièrement des groupes ethniques Talinga, Pakombe, Mbuba, Lese, Humu, Bila et Piri. A l'exception de Piri qui vivent à la fois dans les territoires de Lubero et Beni, les autres sous-groupes ethniques vivent tous dans le territoire de Beni et sont, par conséquent, facilement assimilés et intégrés dans la communauté Nande du Nord-Kivu. Avec l'intégration de ces 7 sous-groupes dans la communauté Nande, il y a lieu de noter que la province du Nord-Kivu est peuplée par 10 groupes ethniques que sont les Nande, les Hutu, les Tutsi, les Nyanga, les Kano, les Hunde, les Tembo, Les Kumu, Les Kusu et les Mbuti (pygmées) ${ }^{64}$.

Ainsi parmi les 10 grands groupes ethniques de la province, 5 seulement sont représentés au sein de l'Assemblée provinciale du NordKivu. Il s'agit des groupes ethniques Nande, Hutu, Hunde, Nyanga et Kusu. Les autres groupes ethniques tels que Tembo, Kano, Kumu, Tutsi et Mbuti n'ont aucune représentation au niveau de l'organe délibérant de la province. La principale raison de cette absence de représentativité est liée au faible poids démographique de ces groupes ethniques au sein de la province. Il

\footnotetext{
${ }^{64}$ Lire pour plus de lumière le Rapport annuel de l'Assemblée Provinciale du Nord-Kivu, décembre 2014. Les informations contenues dans ce rapport ont également été confirmées lors de l'entretien avec Monsieur Stéphane Lupao, secrétaire de la plate-forme « Maraza wa Bazee », Goma, 5 novembre 2014
} 
faudrait, peut-être, attendre de nouvelles législatives provinciales afin d'améliorer leur capacité de mobilisation politique, obtenir des sièges au sein de l'organe délibérant de la province et avoir droits au chapitre lors de traitement de dossiers importants d'intérêt provincial.

Tableau 2 : Liste des députés remplacés au sein de l'Assemblée Provinciale du Nord-Kivu

\begin{tabular}{|c|c|c|c|}
\hline $\begin{array}{l}\text { Circonscription } \\
\text { électorale }\end{array}$ & Député ayant remplacé & Député ayant été remplacé & Motif du remplacement \\
\hline $\begin{array}{l}\text { Territoire de } \\
\text { Beni }\end{array}$ & $\begin{array}{l}\text { Hon. Kasereka Giza } \\
\text { Jean de Dieu }\end{array}$ & $\begin{array}{lll}\text { Hon. } & \text { Nzanzu } & \text { Kasivita } \\
\text { Carly } & & \\
\end{array}$ & $\begin{array}{l}\text { Désigné } \\
\text { provincial }\end{array}$ \\
\hline $\begin{array}{l}\text { Territoire } \\
\text { Lubero }\end{array}$ & $\begin{array}{ll}\text { Hon. } & \text { Maghuta } \\
\text { Kamwenge } & \end{array}$ & $\begin{array}{lr}\text { Hon. } & \text { Vahamwiti } \\
\text { Mukesyarwa } & \text { Jean } \\
\text { Chrysostome } & \end{array}$ & $\begin{array}{l}\text { Appelé à une autre } \\
\text { fonction (ADG) }\end{array}$ \\
\hline $\begin{array}{l}\text { Territoire } \\
\text { Masisi }\end{array}$ & $\begin{array}{l}\text { Hon. Banda Mulera } \\
\text { Bernard }\end{array}$ & $\begin{array}{l}\text { Hon. Mashagiro Nzeyi } \\
\text { Jérôme }\end{array}$ & $\begin{array}{l}\text { Invalidé pour cause de } \\
\text { rébellion }\end{array}$ \\
\hline $\begin{array}{l}\text { Territoire } \\
\text { Rutshuru }\end{array}$ & $\begin{array}{ll}\text { Hon. } & \text { Mizerero } \\
\text { Sebirande } & \text { Déogratias }\end{array}$ & $\begin{array}{l}\text { Hon. Serufuli Ngaya } \\
\text { Baseka Eugène }\end{array}$ & $\begin{array}{l}\text { Appelé à une autre } \\
\text { fonction (ADG) }\end{array}$ \\
\hline Ville de Beni & $\begin{array}{l}\text { Hon. Mayombo Omari } \\
\text { Jean }\end{array}$ & $\begin{array}{l}\text { Hon. Kasereka Kalwaghe } \\
\text { Charles }\end{array}$ & $\begin{array}{ll}\begin{array}{l}\text { Désigné } \\
\text { provincial }\end{array} & \text { ministre } \\
\end{array}$ \\
\hline $\begin{array}{l}\text { Ville } \\
\text { Butembo }\end{array}$ & $\begin{array}{l}\text { Hon. Paluku Kataka } \\
\text { Emery }\end{array}$ & $\begin{array}{l}\text { Hon. Palulu Kahongya } \\
\text { Julien }\end{array}$ & $\begin{array}{l}\text { Elu Gouverneur de } \\
\text { Province le } 27 \text { janvier } \\
2007\end{array}$ \\
\hline Ville de Goma & $\begin{array}{l}\text { Hon. } \\
\text { Kyalondawa } \\
\text { Pierre }\end{array}$ & $\begin{array}{l}\text { Hon. Gachaba Maniraguha } \\
\text { François }\end{array}$ & $\begin{array}{l}\text { Invalidé pour cause de } \\
\text { rébellion }\end{array}$ \\
\hline $\begin{array}{l}\text { Territoire } \\
\text { Walikale }\end{array}$ & $\begin{array}{l}\text { Hon. Eliba } \\
\text { Baudouin }\end{array}$ & $\begin{array}{ll}\text { Hon. Mwami Kabutwa } \\
\text { Djuma Isombya }\end{array}$ & Décédé en 2014 \\
\hline $\begin{array}{l}\text { Territoire } \\
\text { Masisi }\end{array}$ & & $\begin{array}{l}\text { Hon. Mwami Bahati } \\
\text { Kaembe Mapifumo }\end{array}$ & $\begin{array}{l}\text { Décédé le } 19 \text { février } \\
2015\end{array}$ \\
\hline
\end{tabular}

Source : Rapport annuel de l'Assemblée Provinciale du Nord-Kivu, décembre 2014, actualisé

Le présent tableau fait référence aux députés remplacés au cours leur mandat soit pour cause de décès, soit puisque appelés à remplir d'autre fonction au niveau provincial ou national. En effet, le tableau indique qu'au total 9 députés ont été remplacés depuis l'entrée en fonction de l'Assemblée Provinciale du Nord-Kivu en fin 2006. Parmi les députés ayant cédé leurs postes, il s'observe qu'1 député a été élu Gouverneur de province en la personne de l'honorable Julien Paluku Kahongya, 2 députés ont quitté l'Assemblée Provinciale puisque nommés au postes ministériels au niveau provincial, 2 députés ont été appelés à occuper le poste d'administrateur Délégué Général (ADG) au niveau national, 2 députés ont vu leur mandat invalidé pour participation à la rébellion du CNDP, un acte considéré comme trahison et, enfin, 2 députés ont laissé leurs postes pour cause de décès. Avec cette configuration au niveau de l'Assemblée Provinciale du Nord-Kivu, il nous a paru important de porter la même analyse au niveau du gouvernement 
provincial afin d'apprécier le poids politique et stratégique de chaque groupe ethnique en termes de représentativité politique.

\section{Représentativité ethnique au sein du Gouvernement Provincial}

«Selon la constitution de la RDC, le gouvernement provincial est composé d'un Gouverneur, d'un Vice-gouverneur et des ministres provinciaux. Le Gouverneur et le Vice-gouverneur sont élus pour un mandat de cinq ans renouvelable une seule fois par les députés provinciaux au sein ou en dehors de l'Assemblée provinciale. Ils sont investis par ordonnance du Président de la République. Les ministres provinciaux sont désignés par le Gouverneur au sein ou en dehors de l'Assemblée provinciale. La composition du gouvernement provincial tient compte de la représentativité provinciale» $^{65}$.

Conformément à cette loi constitutionnelle, le gouvernement de la province du Nord-Kivu a été constitué par le Gouverneur élu, l'honorable Julien Paluku Kahongya. Il y a lieu de noter que ce gouvernement a connu beaucoup de remaniement mais jusque fin 2014, sa composition se présentait de la manière ici présentée dans le tableau qui suit.

Tableau 3 : Institutions provinciales du Nord-Kivu et leurs animateurs

\begin{tabular}{|c|c|c|c|}
\hline Institutions & Animateurs & Fonctions & $\begin{array}{c}\text { Groupes } \\
\text { ethniques }\end{array}$ \\
\hline \multirow[t]{5}{*}{$\begin{array}{l}\text { Assemblée } \\
\text { Provinciale } \\
\text { (Bureau) }\end{array}$} & $\begin{array}{ll}\text { Hon. } & \text { Jules } \\
\text { Hakizumwami } & \\
\text { Habimana } & \end{array}$ & $\begin{array}{l}\text { Président de l'Assemblée } \\
\text { Provinciale }\end{array}$ & $\begin{array}{l}\text { Hutu } \\
\text { (Rutshuru) }\end{array}$ \\
\hline & Hon. Jean Mathe Sindi & $\begin{array}{lll}\text { Vice-président de la } \\
\text { l'Assemblée Provinciale }\end{array}$ & Nande (Beni) \\
\hline & Hon. Nkuba Kahombo & $\begin{array}{l}\text { Rapporteur de l'Assemblée } \\
\text { Provinciale }\end{array}$ & Hutu (Masisi) \\
\hline & $\begin{array}{l}\text { Hon. Jean Bosco } \\
\text { Sebishimbo Ruguba }\end{array}$ & $\begin{array}{lcc}\text { Rapporteur adjoint } & \text { de } \\
\text { l'Assemblée provinciale } & \\
\end{array}$ & Hutu (Masisi) \\
\hline & $\begin{array}{l}\text { Hon. Vutsapu Ndungo } \\
\text { Hagay }\end{array}$ & $\begin{array}{l}\text { Questeur de l'Assemblée } \\
\text { Provinciale }\end{array}$ & $\begin{array}{l}\text { Nande } \\
\text { (Lubero) }\end{array}$ \\
\hline \multirow[t]{4}{*}{$\begin{array}{l}\text { Gouvernement } \\
\text { Provincial }\end{array}$} & $\begin{array}{l}\text { Hon. Julien Paluku } \\
\text { Kahongya }\end{array}$ & Gouverneur de Province & $\begin{array}{l}\text { Nande } \\
\text { (Lubero) }\end{array}$ \\
\hline & $\begin{array}{ll}\text { Me } & \text { Feller } \\
\text { Lutayichirwa } & \\
\end{array}$ & Vice-Gouverneur de Province & $\begin{array}{l}\text { Hunde } \\
\text { (Masisi) }\end{array}$ \\
\hline & $\begin{array}{l}\text { Dr } \quad \text { Valérien } \\
\text { Mbaluwirandi Kinywa }\end{array}$ & $\begin{array}{l}\text { Ministère provincial de } \\
\text { l'Administration du territoire, } \\
\text { ordre public, affaires } \\
\text { coutumières et reconstruction }\end{array}$ & $\begin{array}{l}\text { Nande } \\
\text { (Lubero) }\end{array}$ \\
\hline & $\begin{array}{l}\text { Mme Adèle Bazizane } \\
\text { Maheshe }\end{array}$ & $\begin{array}{l}\text { Ministre provincial de } \\
\text { l'Education, Jeunesse, Genre } \\
\text { et Enfant }\end{array}$ & $\begin{array}{l}\text { Kumu/Hutu } \\
\text { (Nyiragongo) }\end{array}$ \\
\hline
\end{tabular}

${ }^{65}$ Article 198 de la Constitution de la République Démocratique du Congo du 18 février 2006. 


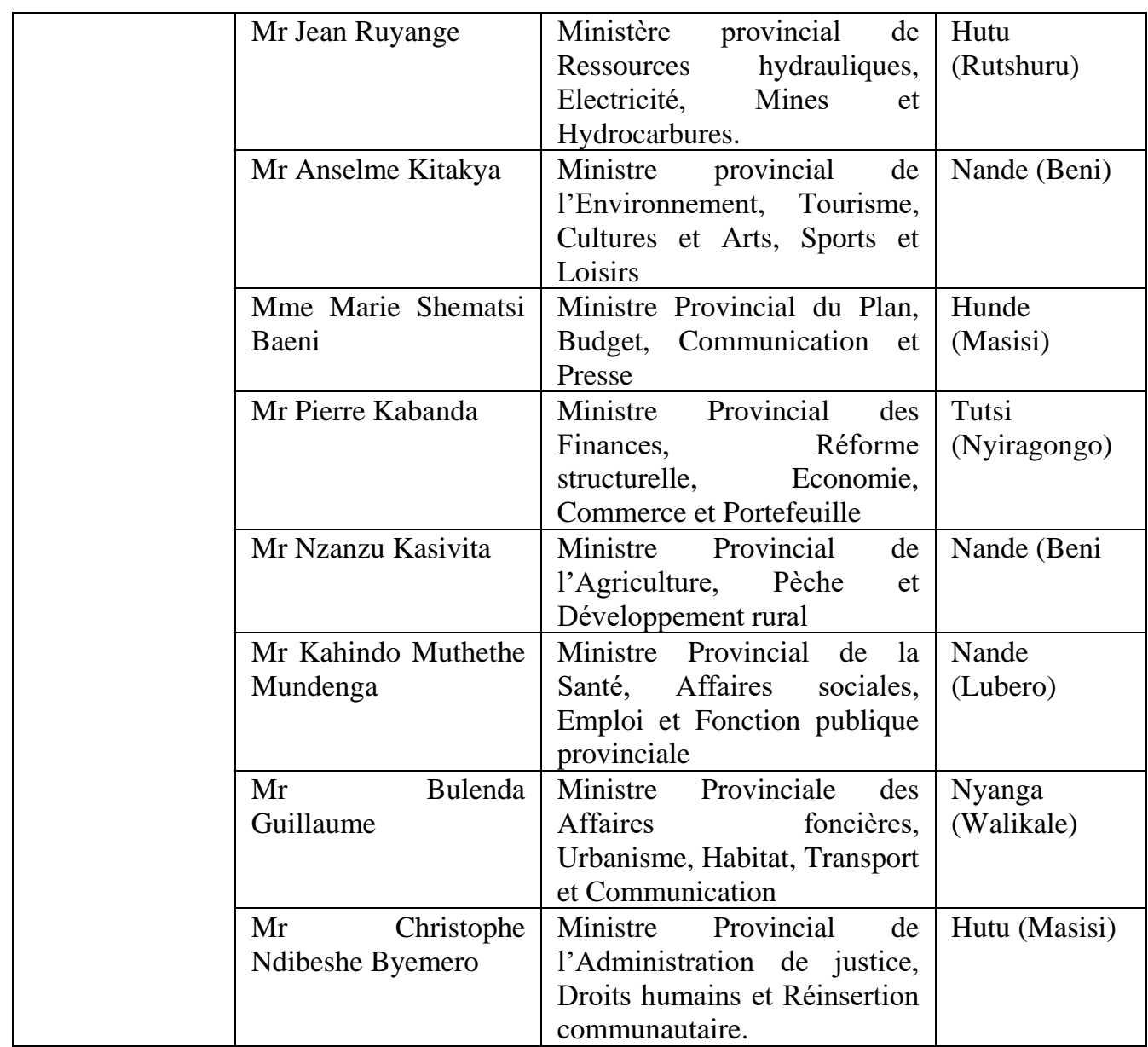

Source : Rapport annuel du Ministère de l'administration du territoire, ordre public, affaires coutumière, exercice 2014

En marge des membres du bureau de l'Assemblée provinciale, le tableau présente les animateurs du gouvernement provincial, leurs groupes ethniques ainsi que leurs territoires d'appartenance. Le gouverneur de province a été élu grâce à la forte représentativité de la communauté Nande au sein de l'Assemble provinciale en tant que corps électoral pour l'élection du gouverneur et du vice-gouverneur au second degré. Le vice-gouverneur, non membre de l'Assemblée provinciale, a été soutenu par la communauté Hunde, troisième force politique au sein de l'Assemblée provinciale étant donné que la communauté Hutu, deuxième force politique de l'Assemblée provinciale, avait déjà brigué le poste du Président de l'Assemblée provinciale.

Parmi les 10 ministres provinciaux, la communauté Yira occupe 4 postes, soit $40 \%$ de représentativité dont 2 pour le territoire de Beni et 2 pour le territoire de Lubero. Ceci sans compter le Gouverneur de province 
également membre de la même communauté. La communauté Hutu compte 2 ministres, soit $20 \%$ de représentativité. Les communautés hunde et Nyanga, Kumu ${ }^{66}$ et Tutsi ${ }^{67}$ comptent respectivement 1 ministre provincial, soit $10 \%$ de représentativité pour chacune. Mais la communauté hunde se trouve également représenté au gouvernement provincial par la personne du vice-gouverneur également membre de cette communauté. A première vue, la logique de représentativité provinciale a été respectée, à l'exception de la communauté tutsi et Kumu qui, sans une représentation provinciale, occupent de postes au sein du gouvernement provincial. Les communautés Tembo, Kano et Mbuti ne sont représentées ni à l'Assemblée provinciale ni au gouvernement provincial du Nord-Kivu de 2006 jusqu'à ce jour.

\section{Discussion}

L'univers politique est un monde compétitif. Les conflits sont de tout genre et éclatent entre acteurs en course pour l'occupation de positions avantageuses. L'Etat, dans cette configuration, doit édicter les règles du jeu auxquelles chaque acteur doit s'y conformer. Pour Philippe Braud, (2011) « Il existe dans le monde de formes des pouvoirs si autoritaires que les gouvernants peuvent, avec succès, empêcher l'expression publique de toute insatisfaction ou tout dissentiment. Dans les régimes à parti unique, l'unanimité de façade aussi bien au sein du parti que dans la consultation électorale, règne sans partage. Avec les moyens différents, les dictatures militaires visent les mêmes résultats en suspendant les organisations politiques et les libertés d'expression. Cette absence d'affrontements visibles ne signifie évidemment pas que les conflits d'intérêts ou de valeurs n'existent pas mais ils demeurent clandestins ou latents. Ce phénomène explique d'ailleurs le paradoxe relevé par Alexis de Tocqueville. Les explosions de mécontentement ne surviennent pas quand les choses vont de mail en pis mais, au contraire, quand les gouvernants autocratiques

\footnotetext{
${ }^{66}$ Pour le représentant de la communauté Kumu au sein du gouvernement provincial, de doute persiste quant à son appartenance à leur communauté du territoire de Nyiragongo. Des informations persistantes font état de son appartenance à la communauté hutu du même territoire et s'est fait passer pour un kumu afin de briguer le poste au niveau du gouvernement provincial. Interrogé à ce sujet, le chef du secteur de Bakumu de Nyiragongo n'a ni nié ni confirmé cette version d'informations. Cependant, au sein des membres de la société civile de Nyiragongo, l'intéressé serait bel et bien membre de la communauté hutu de Nyiragongo.

${ }^{67}$ Non représentée à l'Assemblée provinciale, la communauté tutsi s'est vue représentée au sein du gouvernement provincial en 2009 en application de l'accord du 23 mars 2009. Aux termes de cet accord, la branche armée du CNDP devrait intégrer l'armée congolaise et sa branche politique se transformer en parti politique et lutter pacifiquement pour la conquête du pouvoir au niveau national que provincial.
} 
commencent à entamer de timides réformes, relâchant la pression exercée sur les assujettis ».

Les démocraties pluralistes, au contraire, banalisent en pratique l'idée de conflit. Les libertés d'expression ne peuvent servir qu'à exprimer la pluralité légitime des intérêts et des aspirations dans une société différenciée. De même, les organisations démocratiques acceptent elles-mêmes la compétition pour le renouvellement de leurs dirigeants et, dans le cadre de leurs statuts, la libre expression de différends sur les objectifs à atteindre ou sur les tactiques à mettre en œuvre. Par ailleurs, le processus institutionnel de canalisation et de régulation des conflits sont mis en place (Braud P, 2011). Mais dans la plupart des pays en voie de développement, le processus de canalisation et de régulation des conflits ne sont pas toujours respectés par des acteurs politiques qui n'accordent pas confiance ou légitimité aux dirigeants en place. Dans ce contexte, la recherche de résolution de différends quitte le cadre démocratique et pacifique mis en place pour s'orienter vers la violence. Tel est le cas de la situation qui règne au NordKivu depuis l'organisation des élections de 2006. L'opinion nationale et internationale croyait à la décrispation totale des tensions sociales dans cette province à travers la libération de l'exercice de liberté publique longuement confisqué par le régime Mobutu. Cependant, la conflictualité s'est poursuivie et la province a assisté à l'émergence de nouveaux acteurs armés avec des finalités bien controversées.

En effet depuis le 30 juin 2003, la compétition politique ou la lutte pour la conquête et l'exercice du pouvoir devrait normalement s'effectuer par des moyens pacifiques. Mais la transition politique de 2003 à 2006 était parsemée de moment de trouble ayant pour origine le rapport de forces entre acteurs politiques à l'Est du pays en général et au Nord-Kivu en particulier. Après les élections pluralistes de 2006, plutôt que de s'engager droitement vers la pacification effective du pays et la normalisation de la vie politique, la province du Nord-Kivu est restée marquée par le recours systématique à la violence comme moyen d'expression politique, d'accès au pouvoir et aux ressources économiques. Les divergences se sont rapidement apparues quant à la manière de conduire les affaires politiques dans la province mais surtout au regard de la configuration de l'univers politique de la province où certains groupes ethniques se sont vu non représentés au sein des institutions provinciales.

L'analyse montre que la non représentativité du groupe ethnique tutsi au sein de deux institutions politiques du Nord-Kivu serait l'un des facteurs à la base du recours à la violence après les élections de 2006 au pays. En dépit de l'activisme des FDLR et d'autres groupes d'autodéfense populaire dans la province, la violence était localisée dans les coins les plus reculés et n'avait pas pour cible la conquête et l'exercice du pouvoir politique. C'est à l'issue 
des résultats du second tour de l'élection présidentielle couplée de législatives provinciales et la constitution de gouvernement provincial du Nord-Kivu auxquelles le groupe ethnique tutsi n'était pas représenté que la violence armée s'est accentuée. Frustrés par la non représentativité au sein des institutions politiques provinciales, les acteurs du groupe ethnique tutsi se sont rapidement concertés pour donner naissance au Congrès National pour la Défense du Peuple (CNDP) dont l'un des objectifs principaux était la protection et la défense des intérêts politiques, économiques et existentiels des membres de leur communauté.

Le CNDP constituait ainsi l'outil ou l'instrument de pression politique en vue d'obtenir la participation politique ou, mieux, la représentativité politique du groupe au sein des institutions politiques du Nord-Kivu et au niveau national. Le recours à la violence était ainsi utilisé comme moyen d'expression politique par le groupe pour se faire entendre ou faire passer ses revendications politiques. La conférence pour la paix de Goma en 2008, le mixage des troupes et l'accord du 23 mars 2009 obtenus après l'année 2007 marquée par plusieurs affrontements armés entre le CNDP et 1'armée congolaise, peuvent être considérés comme les retombés politiques de la stratégies adoptée par ce groupe ethnique pour obtenir les avantages politiques, militaires et sécuritaires non seulement au niveau du Nord-Kivu mais aussi sur le plan national. C'est dans cette optique qu'un ministère provincial du Nord-Kivu (finances, commerce, économie et portefeuille) a été confié au représentant de cette communauté conformément à l'accord du 23 mars 2009 signé entre le gouvernement congolais et le Congrès National pour la Défense du Peuple (CNDP) à Goma.

Avec le recours à la violence adopté comme stratégie de lutte politique, les autres groupes ethniques ne sont pas restés inactifs. Nombreux d'entre eux se sont organisés pour répondre à la violence par la violence et utiliser le même langage politique mobilisé par les autres. Ceci explique en partie la prolifération des groupes armés dans la province au lendemain de la création du CNDP et son activisme dans plusieurs territoires au Nord-Kivu. La création du CNDP est à considérer comme l'élément déclencheur de la réactivation des groupes d'autodéfense populaire en sommeil dans la province afin de répondre efficacement à la menace de domination militaire que le mouvement voudrait imposer sur les autres communautés au sein de la province du Nord-Kivu.

Le non-respect de l'accord du 23 mars 2009, côté gouvernement congolais, serait à la base de la défection des militaires CNDP ayant intégré l'armée congolaise aboutissant ainsi à la création, en avril 2012, du Mouvement du 23 mars 2009 communément appelé le M23. Les autres groupes armés de la province s'identifient comme de mouvements communautaires d'autodéfense populaire face à l'activisme récurent du 
CNDP et du M23 mais aussi des FDLR présents et actifs dans plusieurs territoires du Nord-Kivu. Ce rapport de forces entre acteurs politiques qui encouragent les membres de leurs groupes ethniques à recourir aux armes pour se protéger et défendre leurs communautés respectives est également à la base de l'escalade de la violence que connait la province depuis plusieurs années. Frustrés par le non contrôle de la province sur le plan politique, les acteurs politiques de la communauté tutsi se seraient résolus de mettre en place un moyen de pression et une main mise militaire sur la province (contrôle sécuritaire des territoires) afin d'avoir droit au chapitre et se présenter dans des négociations en position de force. Par ailleurs, l'analyse montre que sur le terrain, l'activisme du CNDP sur le champ de la violence au Nord-Kivu serait également lié à la course vers 1'occupation des sites d'exploitation artisanale des minerais et ce, dans le but de parvenir à une domination économique en plus de la domination militaire et sécuritaire dont ils disposent déjà dans la province. Une telle double domination (sécuritaire et économique) leur permettrait de détenir incontestablement le leadership sur l'ensemble de la province du Nord-Kivu voire au-delà, bien que non ou moins représentés au sein des institutions politiques.

En définitive, il se dégage que la question de représentativité de groupes ethniques constitue l'un des enjeux dans la lutte politique au NordKivu. Les rapports de forces politiques ayant dégénéré, au fil des ans, aux affrontements armés entre groupes ethniques, l'entretien et le maintien des groupes armés devient un enjeu capital pour s'affirmer sur le plan politique, se faire attendre et d'assurer la représentativité au sein des institutions politiques provinciales. En conséquence, chaque groupe ethnique tend à construire son groupe armé destiné à protéger la communauté en cas d'attaque ennemi. Une manière de dissuader l'adversaire et le décourager à s'attaquer au groupe visé car celui-ci dispose d'un moyen de défense approprié. Une forme de privatisation de la sécurité collective par recours à la violence armée vu l'absence prolongée de l'Etat qui devrait jouer ce rôle. Cette configuration du paysage politique contribue également, dans une certaine mesure, à la prolifération des groupes armés qui sèment désordre et désolation parmi les populations civiles au Nord-Kivu.

\section{Conclusion}

L'analyse effectuée dans le cadre de cette réflexion atteste que depuis les années 1990, la République Démocratique du Congo est pratiquement tournée vers la représentation des diversités géographiques et ethniques. La représentation de l'unité du groupe national, chère au Président Mobutu, n'est plus qu'un souvenir lointain. A cette époque, chaque citoyen congolais avait la possibilité de briguer un poste politique partout sur le territoire national en fonction de ses capacités, compétences et popularités et ce, sans 
référence à son appartenance géographique, ethnique ou clanique. Il n'en est plus le cas aujourd'hui. La province du Nord-Kivu est reconnue comme celle où le particularisme ethnique est très prononcé. La question de l'ethnicité reste au cœur des débats et tractations politiques au niveau local et donne souvent lieu aux affrontements armés qui endeuillent quotidiennement cette partie de la République. En plus de l'économie de guerre, de l'accès à la terre et de nationalité des immigrés comme facteurs de la persistance des conflits armés dans la région, il importe de mentionner l'épineuse question de représentativité des groupes ethniques au sein des institutions politiques provinciales depuis que l'accès autorité au pouvoir passe par des élections. Les législatives nationales et provinciales organisées en 2006 ont eu pour effet la non représentativité du groupe ethnique tutsi à l'Assemblée Nationale mais aussi Provinciale du Nord-Kivu, lieu de discussions sur les principales questions d'intérêt provincial.

Cette nouvelle donne politique a frustré le groupe concerné qui s'est senti vulnérable au risque de perdre les acquis politiques et économiques obtenus pendant la guerre de l'AFDL et du RCD. Pour ce faire, la construction d'un nouveau groupe armé grâce aux officiers militaires et autres troupes restés non intégrés a été réalisée. Le CNDP, groupe armé à dominance tutsi, a été créé pour défendre les intérêts du groupe ethnique tutsi considérés comme menacés. Pour ce groupe ethnique, il fallait recourir aux armes pour exiger leur représentativité politique. En conséquence, et se sentant menacés à leur tour, les autres groupes ethniques de la province (Nande, Hutu, Tembo, Hunde, Nyanga, Kano,...) ont résolu d'en faire autant afin de se protéger et utiliser la même stratégie politique devenu mode opératoire des acteurs de la région.

La conférence pour la paix de Goma en 2008, le mixage des troupes et l'accord du 23 mars 2009 peuvent être considérés comme les retombés politiques de la stratégies adoptée par ce groupe ethnique pour obtenir les avantages politiques, militaires et sécuritaires non seulement au Nord-Kivu mais aussi sur le plan national. Si, on niveau provincial les acquis ont été visibles (obtention d'un ministère provincial, nomination au sein de l'administration publique et surtout au sein de l'armée), il n'a pas été possible d'obtenir des ministères au niveau national faute de représentativité à l'Assemblée Nationale. La non réalisation de cette exigence, associé à celle de retour des réfugiés tutsi congolais encore présents au Rwanda et en Ouganda, ont été les mobiles avancés par ce groupe ethnique pour justifier la création du M23. Le gouvernement congolais devra prendre en compte l'ensemble des facteurs ci-haut évoqués dans le traitement et la recherche de solution durable à la persistance des conflits armés au Nord-Kivu et sur l'ensemble de la partie Est du pays. 


\section{References:}

1. Amselle, JL et Elikia Mbokolo (dirs.),. (1985), Au cour de l'ethnie, Paris, La Découverte.

2. Amuri Misako, FD. (2011), Symbolisme de la légitimation de la violence chez les milices «mai-mai» du Maniema (CongoKinshasa): continuité et réinvention du messianisme nationaliste, Thèse de doctorat en science politique, UNIKIS, FSSAP.

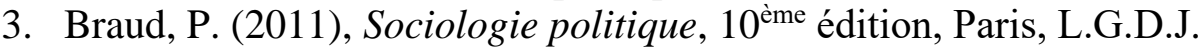

4. De Saint Moulin, L. (Juin 2006), RDC, Les principaux défis de l'après élections, CEPAS (membre du Centre AVEC), Kinshasa.

5. Hermet, G., Badie, B., Birbhaum, P., Braud, P. (2010), Dictionnaire de la science politique et des institutions politiques, $7^{\mathrm{ème}}$ édition revue et augmentée, Armand Colin, Paris.

6. Human Rights Watch. (1997), Rapport annuel sur la République Démocratique du Congo.

7. Lissendja Bahama, T. (Mars 2010), Mobilisation électorale et participation populaire aux élections de 2006 à Kisangani: stratégies des acteurs, Mémoire d'Etudes Supérieurs (DES) en science politique, FSSAP, SPA, UNIKIS.

8. Mashaury Kule Tambile. (Décembre 1987), « Migrations internationales et problèmes d'intégration. La question rwandaise au Zaïre », Presse Universitaire de Kisangani, Revue de l'IRSA $n^{\circ} 2$.

9. Mathieu, P., et Kazadi, C. (1990), «Quelques aspects des législations et pratiques foncières au Zaïre: ambiguïtés et enjeux pour les politiques agricoles ». Mondes en Développement, vol.18, ${ }^{\circ} 69$.

10. NKoko Lipambala, J. (2009), La transformation des groupes armés en partis politiques. Expérience du Rassemblement Congolais pour la Démocratie en République Démocratique $d u$ Congo, Mémoire d'Etudes Supérieures en SPA, FSSAP, UNIKIS, inédit.

11. Pabanel, J-P. (1991), «La question de nationalité au Kivu », Politique Africaine $\mathrm{n}^{\circ} 41$.

12. Pourtier, R. (19997), « Du Zaïre au Congo : un territoire en quête d'Etat », Afrique contemporaine, $\mathrm{n}^{\circ} 183$.

13. Pourtier, R. (Mars-Avril1999), « La Guerre des Grands-Lacs », Cahiers français, $\mathrm{n}^{\circ} 290$.

14. Rusamira, E. (2003), « La dynamique des conflits ethniques au NordKivu ; une réflexion prospective », Afrique contemporaine, n²07.

15. Verhaegen, B. (1969), «Les rébellions au Congo », in Les études $d u$ CRIPS, tome I, Bruxelles/Léopoldville, CRIPS-IRES-INEP.

16. Vlassenroot, Koen. et Raeymaekers. (2004), Conflict and social transformation in Eastern DR Congo, Gent, Academic Press. 